\title{
Stereospatial masking and aftereffect with normal and transformed random-dot patterns*
}

\author{
NIGEL LONG and RAY OVER $\dagger$ \\ University of Queensland, St. Lucia 406 7, Australia
}

\begin{abstract}
Masking and aftereffect in the perception of binocular depth were studied using random-dot sterograms as adaptation and target stimuli. Detection of the target was impaired by prior adaptation only when the two stimuli differed in disparity by less than 2 minarc. The masking function was unaffected by uniocular enlargement and blurring within the adaptation stimulus, but masking was no longer selective to disparity when the elements seen by the two eyes were reversed in brightness. The stereoscopic depth aftereffect was also insensitive to uniocular enlargement and blurring, and could not be generated when there was brightness complementation within the adaptation stimulus. Both the masking and aftereffect data are interpreted as evidence that stereospatial detectors in human vision are insensitive to transforms that maintain luminance-spatial correlations in binocular input.
\end{abstract}

Masking paradigms establish the extent to which exposure to an adaptation stimulus impairs detection of a target stimulus. In the perception of contour orientation (Houlihan \& Sekuler, 1968), image motion (Pantle \& Sekuler, 1969), and spatial frequency (Blakemore \& Campbell, 1969), the visibility of the target is maximally impaired when the adaptation and target stimuli are identical in spatial value and masking is progressively weaker as the spatial difference increases. Claims that masking functions provide information about feature detection in human vision (e.g., Blakemore \& Campbell, 1969; Weisstein, 1969) rely on the argument that exposure to the adaptation stimulus renders specific neural detectors inactive for a period of time afterwards. If detectors normally engaged in signaling the target stimulus are now in an adapted state, the signal-noise ratio that is critical for detection can be maintained only by an increase in the energy level of the target stimulus. In these terms, psychophysical data provide an index of the breadth of tuning of neural detectors, because exposure to one stimulus is able to impair detection of another stimulus only to the extent the two stimuli are represented by common neural units.

In Experiments I and II, random-dot stereograms are used as adaptation and target stimuli to study stereospatial masking. A figure-in-depth is seen when the two eyes simultaneously view separate random-dot patterns which are identical except that a group of elements has been shifted laterally in spatial position to one eye relative to the other. On binocular fusion, the translated elements appear clustered as a cohesive figure in front of or behind (depending on the direction of image difference between the two eyes) the unshifted

*This research was supported in part by an award from the Australian Research Grants Committee to Ray Over.

tRequests for reprints should be sent to Ray Over, Department of Psychology, University of Queensland, St. Lucia 4067, Australia. surround. Space perception studied with random-dot stereograms must reflect the response of neural mechanisms tuned to binocular disparity in that neither figure nor depth is visible when the patterns are viewed monocularly (see Julesz, 1971).

Disparity-selective masking has received previous attention in two contexts. Richards (1972) has shown that the apparent depth of an above-threshold binocular target can be shifted (relative to the fixation point) as a function of the disparity value of simultaneously presented surround objects. He attributed this distortion to inhibitory interaction between sets of neural detectors with differential tuning to binocular disparity. This account is analogous to the explanation offered by Blakemore, Carpenter, and Georgeson (1970) of the orientation illusion in which two lines appear shifted from each other in tilt when they are joined to form an acute angle. Both distortions are more appropriately considered as illusions than as masking, which involves obliteration of the target by the adaptation stimulus. Disparity masking was, however, measured in two recent studies in which Blakemore and Hague (1972) and Felton, Richards, and Smith (1972) exposed Ss to a binocularly viewed grating and then determined the threshold for detection of a binocular grating seen in the same or in a different visual plane. In both cases, masking was greatest when the adaptation and target gratings were seen in the same visual plane. Blakemore and Hague found that masking was selective to disparity over a range of \pm 12 minarc, whereas Felton, Richards, and Smith report a tuning range of $\pm 30-45$ minarc.

Disparity-selective masking should be considered in relation to the distinction between fine and coarse stereopsis (see Bishop \& Henry, 1971). Fine stereopsis involves a highly specific pattern-matching process based on local features in the images of the two eyes, whereas with coarse stereopsis depth perception can occur with 


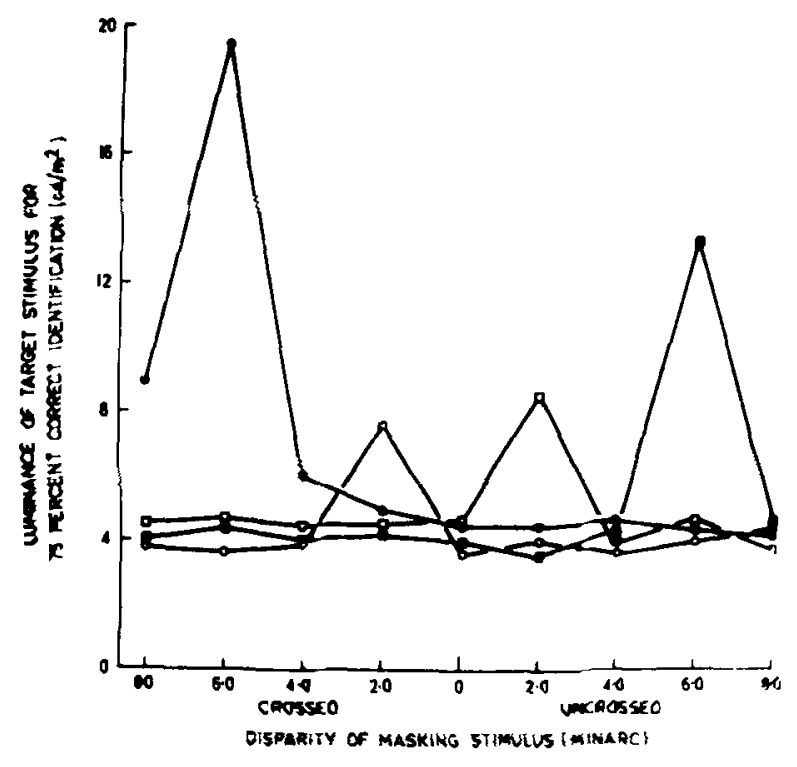

Fig. 1. Thresholds for stereoscopic depth as a function of adaptation and target disparity. Parameter is target disparity: $(0-\infty) 2$ minarc crossed; $(\mathrm{a}-\longrightarrow$ ) 2 minarc uncrossed; (๑-—) 6 minarc crossed; (-——) 6 mintarc uncrossed.

binocular inputs dissimilar in form, luminance, and contrast, and differing in disparity by several degrees. Depth perception with random-dot stereograms is never accompanied by double images, and it therefore involves only fine stereopsis. The same limitation does not apply with the displays used by Blakemore and Hague (1972) and Felton, Richards, and Smith (1972). For this reason, Experiment 1 measured disparity-selective masking in fine stereopsis. The threshold for detection of depth in a target random-dot stereogram was established as a function of the extent of binocular disparity present in the adaptation random-dot stereogram shown immediately beforehand.

\section{EXPERIMENT I}

\section{Method}

Subjects. The five Ss had normal or corrected-to-normal vision, and they were experienced in the perception of random-dot stereograms at brief exposures.

Materials and Procedure. A Gerbrands three-field tachistoscope (Mode! T-3B-1) was used to display the adaptation and target stereograms in succession, with each field of a stereopair projected to the appropriate eye by separate pairs of Polaroid filters set parallel to each other. Two prisms (4.25 diopters) were inserted into a modified eyepiece of the tachistoscope to aid binocular fusion, and there was also a fixation point viewed by both eyes and centrally located in the same visual plane as the background.

A set of stereostimuli, prepared from computer-generated equaliy probabie black and white dots, was used 10 manipulate the visual depth of a $2 \times 2$ deg center square within the adaptation and target displays. In the basic stereopair (zero disparity), the left field (Lf) and the right field ( $R f)$ consisted of identical matrices of $960 \times 960$ randomly positioned dots, which on fusion yielded a binocular field subtending 5 deg 20 minare vertically and horizontally. All other stereopairs were derived from this basic pair by horizontal translation of a center square of elements within $\mathrm{Lf}$ and $\mathrm{Rf}$. The range from 8 minarc crossed disparity to 8 minarc uncrossed disparity in 2-minarc steps was uscd, and the stereostimuli were identical in all other respects,

Oisparity masking was measured using a blockwise tracking method (Over, Broerse, \& Crassini, 1972). On each trial, the fiisation point was shown for $1 \mathrm{sec}$ prior to presentation for $500 \mathrm{msec}$ of the adaptation stereogram (space-average luminance, $18.0 \mathrm{~cd} / \mathrm{m}^{2}$ ). Following a dark interval of $10 \mathrm{msec}, \mathrm{S}$ viewed for a fixed period (90 to $120 \mathrm{msec}$, depending on S's stereoacuity as measured in preliminary testing) the target stereogram. His task was to report whether the target display had contained disparity information, and, as stereoscopic depth is seen pror to figure (Oves \& Long, 1973), the judgment was in effect one of whether depth was visible. On some test trials, the target stereogram did not contain disparity information, and in other cases, disparity ( 2 minarc and 6 minarc of crossed and uncrossed disparity) was present. There were nine disparity levels ( 0 disparity and $2,4,6$, and 8 minarc of crossed and uncrossed disparity) within the adaptation stereogram. The threshold for detection of stereoscopic depth was measured for each $S$ under the 36 factorial combinations of adaptation and target disparity, with the sequence in which the combinations were tested randomized between $S s$.

Within each disparity combination, the target stereogram was shown at a fixed space-average luminance over a block of 12 trials. On 6 of these trials, the stereogram did not contain depth information, and on the other 6 trials, the appropriate disparity value was shown. The $S^{t} s$ task was to differentiate these two types of display. The space-average luminance of the target stereogram was increased over biocks of 12 mals in logatithmic sleps if $\mathrm{S}$ was less than $75 \%$ accurate in judgments on a block, or decreased if accuracy exceeded $75 \%$. This procedure continued until $75 \%$ accuracy was attained over a block or bracketed by successive blocks. There was a 10-sec dark interval between successive trials in a block.

\section{Results}

The threshold for stereoscopic detection at each combination of the adaptation and test conditions was the space-average luminance at which $\mathrm{S}$ attained $75 \%$ accuracy in differentiating target presentations providing disparity information from the 0-disparity "catch" trials. This value was determined by extrapolation wherever necessary. The threshold luminances obtained when the adaptation stereogram did not provide disparity information serve as the baselines against which impairment in detection attributable to the stereospatial properties of the adaptation stimuli can be established. Masking is in evidence whenever the luminance level required for $75 \%$ accuracy in detection is above the relevant baseline. Figure 1 shows the thresholds for detection of each of the four test stimuli ( 2 minarc and 6 minarc of crossed and uncrossed disparity) as a function of the direction and amount of disaprity within the adaptation stereogram.

It is clear from inspection of Fig. 1 that detection of the test stimulus was impaired only when $S$ had previously viewed an adaptation stereogram that contained the same depth information as the test stimulus. In an analysis of variance based on these data, the direction of disparity within the adaptation stimulus was examined as a factor by disregarding measures 
obtained under 0-disparity adaptation. Threshold luminance varied significantly as a function of the amount (ignoring direction) of disparity within the test stimulus, $F(1,4)=7.43, p<.05$, and within the adaptation stimulus, $F(4,16)=8.97, p<.01$. The direction of disparity (crossed or uncrossed) within the test stimulus, $F(1,4)=1.01, p>.05$, and within the adaptation stimulus, $F(1,4)=4.06, p>.05$, did not exert significant influence over threshold luminance. The interaction between the direction of disparity within the adaptation and test stimuli was significant, $F(1,4)=$ $22.84, p<.01$, as was their combined interaction with test disparity, $F(1,4)=33.12, p<.01$, and adaptation disparity, $F(4,16)=9.35, p<.01$. The four-way interaction between the amount and direction of disparity within the adaptation and test stimuli was also significant, $F(4,16)=15.94, p<.01$. None of the remaining interactions was significant.

Multiple comparison of means through Duncan's test showed that the visibility of a binocular target was maximally impaired by prior exposure to another stereospatial stimulus that contained the same disparity information. Stereoscopic masking was highly specific in that adaptation had no influence on detection, except around 6 minarc crossed disparity, when the successively presented binocular stimuli differed in disparity by 2 minarc or more. This range is far smaller than the 12-minarc (Blakemore \& Hague, 1972) and the 30-45-minarc (Felton, Richards, \& Smith, 1972) values that have been obtained with binocularly viewed gratings.

\section{EXPERIMENT II}

Stereoscopic vision is controlled by binocular variables additional to spatial disparity Julesz (1964), using random-dot patterns, found that uniocular blurring and image magnification result in little or no impairment in depth perception, but stereopsis is lost when there is reversal in theluminance relationships of elements between the two eyes. These data suggest that disparity-analyzing mechanisms in the visual system tolerate certain degradations of stereoscopic information but are rendered inactive by other transforms. The masking paradigm allows indirect study of this issue on the grounds that a target is less visible following exposure to the adaptation stimulus only to the extent that both stimuli are normally signaled by the same neural units. Within this framework, the masking functions reported for stereoscopic vision in Experiment I should be unaffected by uniocular blurring and magnification within the adaptation stereogram, but complementation in brightness between the adaptation stereopair should result in loss of disparity-specific masking. This question is examined in Experiment II.

\section{Method}

Disparity selectivity in masking was studied by maintaining a fixed level of disparity (6 minarc crossed disparity) within the adaptation stimulus and measuring thresholds for detection of targets of $2,4,6$, and 8 minarc crossed disparity by the procedures described for Experiment I. These measures were compared with threshold values obtained when a 0 -disparity stereogram was shown in place of the adaptation stimulus. Masking due to exposure to the adaptation stimulus was indexed by impairment in detection of a target relative to this baseline.

The adaptation and target stereograms were matrices of $960 \mathrm{x}$ 960 randomly positioned black and white dots that yielded a binocular display subtending 5 deg horizontally and vertically. In the case of the target stimuli and one of the adaptation conditions (normal), the random-dot patterns seen by the two eyes differed solely in the relative position of a 2 -deg square of elements located in the center of the binocular field, and this variable determined the disparity within the display. The other three adaptation conditions differed from the normal condition such that in one case the luminance of the black and white elements was reversed between the two eyes (complementation), in another the elements seen by the right eye were magnified by $10 \%$ (enlargement), and in a further case the elements seen by the right eye were blurred (blurring). The space-average luminance of each adaptation stimulus was maintained at $18.0 \mathrm{~cd} / \mathrm{m}^{2}$.

Masking was studied by the procedures described for Experiment I, with $S$ required to report on each trial whether a target stereogram containing disparity information or a zero-disparity "catch" stimulus had been shown. The luminance of the display on the first block of 12 trials given under each condition was $9.50 \mathrm{~cd} / \mathrm{m}^{2}$, and this value was increased or decreased in logarithmic steps until $75 \%$ accuracy was achieved in a block or bracketed over successive blocks. Baseline measures were initially gained from the four Ss by measuring detection of the four target disparities when the 0-disparity stereogram was shown in place of the adaptation stimuli. Sixteen threshold measures, representing the factorial combinations of the four adaptation and the four target values, were then obtained from each $S$. The sequence of testing these combinations was varied between Ss by a random order.

\section{Results}

Figure 2 shows the mean space-average luminances required for $75 \%$ accuracy in detection of the four target stimuli $(2,4,6$, and 8 minarc crossed disparity) under the four adaptation conditions (normal, complemented, enlarged, blurred) and when a 0-disparity target was shown in place of the adaptation stimuli (baseline condition). There was 6 minarc crossed disparity within each adaptation stereogram. An analysis of variance showed that detection varied significantly as a function of the disparity within the target stimulus, $F(3,21)=$ $18.19, \mathrm{p}<.01$, but did not differ across the adaptation and baseline conditions, $F(4,28)=.67, p>.05$. The interaction between target disparity and adaptation/baseline condition was significant, $F(12,84)$ $=8.27, \mathrm{p}<.01$.

Comparisons between the two-way interaction means by Duncan's multiple range test indicated that disparity-specific masking resulted only with the normal, enlarged, and blurred adaptation stimuli. In these cases, the detectability of the target differed from baseline measures only when the adaptation and target stimuli were identical in disparity. This masking range of \pm 2 minarc is identical to the value obtained in Experiment I. Masking was as pronounced with enlarged 


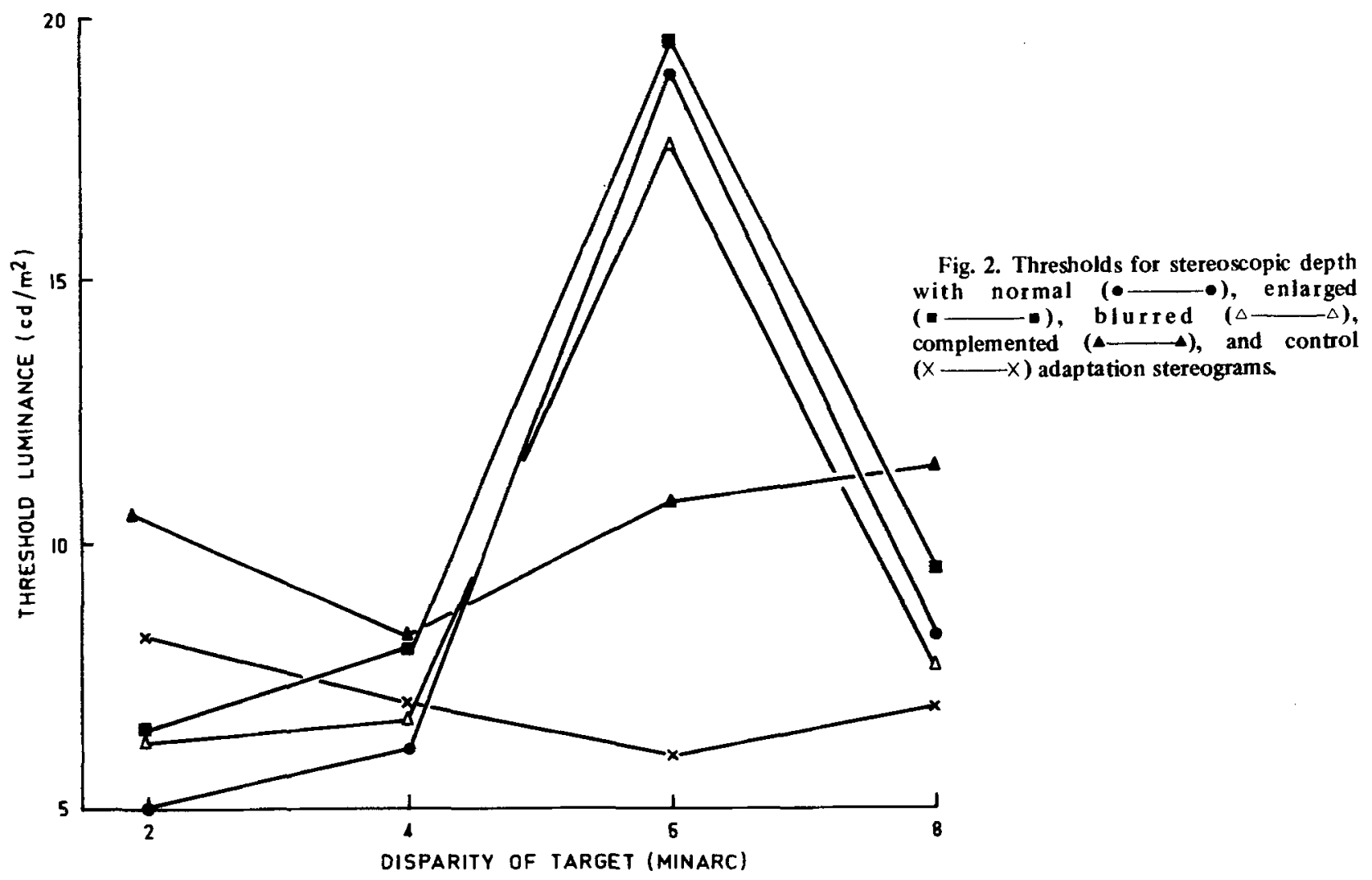

and blurred adaptation stereograms as with the normal adaptation stimulus, and this result is consistent with evidence obtained outside the masking context (see Julesz, 1971) that uniocular enlargement and blurring have little or no effect on stereopsis. The complemented adaptation stereogram did not yield disparity-selective masking. Although detection was poorer than the baseline measures at each target value, the degree of impairment did not differ significantly as a function of the difference in disparity between the adaptation and target stimuli.

\section{EXPERIMENT III}

Successive presentation of random-dot stereograms containing different disparity information produces an aftereffect in binocular depth perception (Blakemore \& Julesz, 1971; Long \& Over, 1973; Over, Long, \& Lovegrove, 1973). The aftereffect takes the form of spatial repulsion, with the binocular target appearing nearer to $S$ following exposure to uncrossed disparity and farther away following exposure to crossed disparity.

Spatial aftereffects have been attributed to selective adaptation of finely tuned neural detectors (see Coltheart, 1971; Over, 1971). The assumption is that the perceived depth of a stereoscopic target is given by the relative activity of detectors maximally responsive to crossed and uncrossed disparity. Thus, detectors sensitive to crossed disparity are most active when a near stimulus is viewd, detectors tuned to uncrossed disparity mainly signal a far stimulus, and a midvalue is represented by a balance between the opposed systems. The further assumption is that neural channels active during inspection are in an adapted state immediately afterwards. Detectors tuned to crossed disparity would be adapted by exposure to a near stimulus, and for a period of time after inspection, the midposition would be represented mainly by the unadapted uncrossed disparity system. Following inspection, the target would therefore appear displaced in depth away from $S$. Adaptation is nonselective when the inspection and target stereostimuli are both presented at the midposition, and for this reason, the apparent depth of the target is not shifted following inspection.

Experiment III measures the stereoscopic depth aftereffect using normal, complemented, enlarged, and blurred stereograms as the adaptation stimuli. The results of Experiment II and the approach to aftereffects outlined above suggest that an aftereffect will not be induced with the complemented adaptation stimuli, while uniocular enlargement and blurring should not result in a smaller aftereffect than found with the normal adaptation stimulus.

\section{Method}

The stimulus displays differed from Experiment II solely in that the target stereograms covered the range from 5 minarc 
Fig. 3. Percentage frequencies with which target squares of different disparities are judged as being behind the surround after exposure to normal $(\bullet-\bullet)$, enlarged $(\bullet-\bullet), \quad$ blurred $(\triangle \longrightarrow \Delta)$, complemented (A- $\wedge)$, and control $(X-X)$ adaptation stereograms.

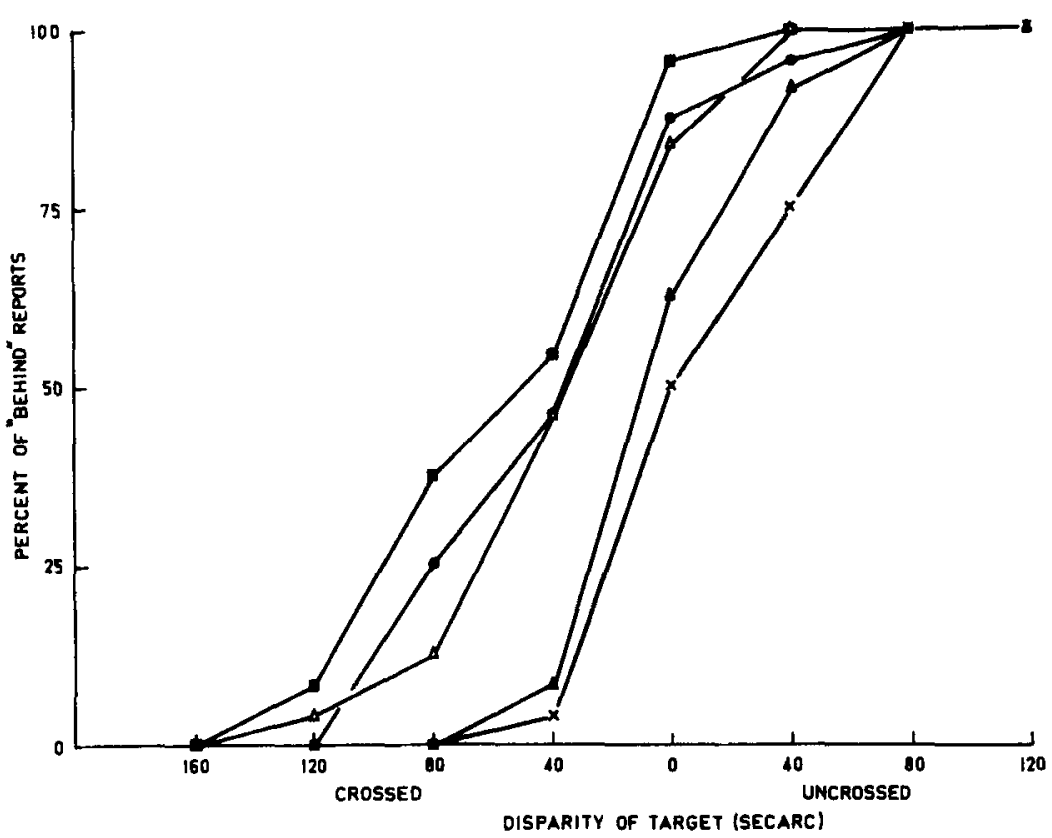

crossed disparity to 5 minarc uncrossed disparity in 40-secarc steps, with the random-dot patterns seen by the two eyes identical in respects other than the disparity value of the center 2-deg square of elements. In the four adaptation stereograms (normal, complemented, enlarged, blurred), the center square was produced by 6 minare crossed disparity, and there was also a baseline condition in which a 0 -disparity stereogram was shown in place of the adaptation stimulus. The space-average luminance of the adaptation, baseline, and target stereograms was held constant at $18.0 \mathrm{~cd} / \mathrm{m}^{2}$.

Each of the four Ss was initially tested under the baseline condition, and a Latin square was employed to decide the sequence in which measures were taken under the adaptation conditions. The aftereffect was measured by the cancellation method described by Long and Over (1973). On each trial, a fixation point was shown for $1 \mathrm{sec}$, then the adaptation stimulus for $500 \mathrm{msec}$, followed, after a 10 -msec dark interval, by display of the target stimulus for $500 \mathrm{msec}$. The S's task was to report on each trial whether the plane of depth of the center square in the target stimulus was in front of or behind its surrounds. Under a given adaptation condition, the target stimulus initially contained 0 disparity, and disparity was varied over successive trials in 40-secarc steps in accord with double-random staircase procedures, until six reversals in judgment had occurred. There was a rest period of $20 \mathrm{sec}$ between trials, and $S$ initiated each trial by operating a hand switch.

\section{Results}

Figure 3 shows the frequency with which the target square, at its different disparity values, was judged as being behind its surrounds after exposure to the baseline and adaptation conditions. It is obvious that the functions obtained with normal, enlarged, and blurred adaptation stimuli are shifted in the opposite direction to the visual depth of the center square during adaptation relative to the functions found with the complemented adaptation stereogram and the 0-disparity baseline condition. For purposes of statistical analysis, the location at which the target square appeared in the same visual plane as its surrounds was calculated under the baseline and adaptation conditions as the disparity midway between values on which $S$ reversed depth judgments. An analysis of variance showed that these measures differed significantly between the five conditions, $F(4,12)=9.34, p<.01$. Comparisons between means showed that the perceived location of the target under the complemented adaptation conditions and the 0-disparity baseline did not differ significantly. The measures under normal, enlarged, and blurred conditions were similar to each other, and differed significantly from the complemented and baseline values.

\section{GENERAL DISCUSSION}

The rationale underlying studies of spatial masking is that exposure to one pattern can impair detection of another pattern only to the extent that the two stimuli are processed in the visual system by the same neural units (see Blakemore \& Campbell, 1969; Blakemore \& Hague, 1972). Within this logic, the masking functions obtained in Experiments $I$ and II indicate that the disparity-selectivity of detectors mediating stereoscopic depth perception with random-dot patterns extends over a range of less than \pm 2 minarc. This value is appreciably less than the disparity ranges of \pm 12 minarc (Blakemore \& Hague, 1972) and \pm 30 to 45 minarc (Felton, Richards, \& Smith, 1972) obtained in masking experiments in which binocularly viewed gratings in the same or in different visual planes were used as adaptation and target stimuli. It was suggested earlier that the latter estimates bear on the tuning of neural mechanisms mediating coarse stereopsis, while the use of random-dot patterns to provide depth information in the present experiments has yielded data specific to fine stereopsis. Bishop and 
Henry (1971) have discussed both the psychophysical and electrophysiological basis for this distinction.

The tuning range of \pm 2 minarc for disparity detectors mediating fine stereopsis is considerably larger than the 2-secarc threshold for stereoscopic acuity reported by Julesz (1971). However, acuity is not necessarily dependent on the breadth of response of detectors. Fine stereoscopic discrimination is possible if visual depth is signaled by the relative activity levels of broadly tuned channels with overlapping sensitivities to disparity and not simply by the response of a single narrowly tuned channel (see Erickson, 1969).

In Experiments II and III, stereoscopic masking and aftereffect remained selective to the disparity relationship between the adaptation and target stimuli when there was uniocular magnification and blurring within the adaptation stimulus, but the selectivity to disparity was lost when the adaptation stereopair were opposite in brightness. Julesz (1964) has shown outside the selective adaptation context that complementation destroys stereopsis while uniocular magnification and blurring produce no impairment. Such data suggest that stereospatial detectors in human vision respond to luminance-spatial correlations between the inputs of the two eyes, with the tuning of a detector dependent on the disparity range over which connectivity analysis occurs. In these terms, the neural mechanisms mediating stereospatial perception are insensitive to binocular transforms that leave luminance-spatial correlations unaltered, but are rendered inactive or noisy by variables that remove these relationships. Within this framework, exposure to the complemented stereogram did not result in disparity-selective masking, as this display did not adapt the detectors by which the visual depth of the target stimulus was represented.

There has been extensive electrophysiological study of the effect of disparity on the responseof binocularly driven cells (see Bishop \& Henry, 1971). Limited attention has been given to binocular variables other than disparity. In one such study, Burns and Pritchard (1968) found that cells in the cat visual cortex lose their selectivity to disparity when thepatterns viewed by the two eyes are opposite in brightness. The expectation from the present data is that binocularly driven cells would maintain their selectivity to disparity under conditions of uniocular blurring and enlargement.

\section{REFERENCES}

Bishop, P. O., \& Henry, G. H. Spatial vision. Annual Review of Psychology, 1971.22, 119-160.

Blakemore. C.. \& Campbell. F. W. On the existence of neurones in the human visual system selectivly sensitive to the orientation and size of retinal images. Joumal of Physiology, $1969,203,237-260$.

Blakemore, C.., Carpenter, R. H. S., \& Georgeson, M. A. Lateral inhibition between orientation detectors in the human visual sy stem. Nature, 1970, 228, 37-39.

Blakemore, C., \& Hague, B. Evidence for disparity detecting neurones in the human visual system. Joumal of Physiology, $1972,225,437-455$.

Blakemore, C.. \& Julesz, B. Stereoscopic depth aftereffect produced without monocular cues. Science, 1971, 171, 286-288.

Burns, B. D., \& Pritchard, R. Cortical conditions for fused binocular vision. Joumal of Phy siology, $1968,197,149-171$.

Coltheart. $M$. Visual feature-analyzers and aftereffects of tilt and curvature. Psy chological R eview, 1971, 78, 114-121.

Erickson, R. P. Stimulus coding in topographic and nontopographic afferent modalities: On the significance of the activity of individual sensory neurons. Psychological Review, activity of individual

Felton, T. B.. Richards, W., \& Smith, R. A. Disparity processing of spatial frequencies in man. Joumal of Physiology, 1972,

$225,349-362$
Houlihan, K., \& Sekuler, R. W. Contour interactions in visual masking. Joumal of Experimental Psychology, 1968, 77, 281-285.

Julesz, B. Binocular depth perception without familiarity cues. Science, 1964, 145, 356-362.

Julesz, B. Foundations of cyclopean perception. Chicago: University of Chicago Press, 1971.

Long. N.. \& Over, R. Stereoscopic depth aftereffects with random-dot patterns. Vision Research, 1973, 13, 1283-1287.

Over, R. Comparison of normalization theory and neural enhancement explanations of negative aftereffects. Psychological Bulletin, 1971, 75, 225-243,

Over, R., Broerse, J., \& Crassini, B. Orientation illusion and masking in central and peripheral vision. Journal of Experimental Psy chology, 1972, 96, 25-31.

Over, R., \& Long, N. Depth is seen before figure with random-dot stereograms. Vision Research, 1973, 13, 1207-1209.

Over, R., Long. N., \& Lovegrove, W. J. Absence of binocular interaction between spatial and color attributes of visual stimuli. Perception \& Psy chophysics, 1973, 13,534-540.

Pantle, A., \& Sekuler, R. Contrast response of human visual mechanisms sensitive to orientation and direction of motion. Vision Research, 1969, 9, 397-406.

Richards, W. Disparity masking. Vision Research, 1972, 12, 1113-1124.

Weisstein. N. What the frog's eye tells the human brain: Single cell analyzers in the human visual system. Psychological Bulletin, 1969, 72, 157-176.

(Received for publication May 14, 1973; revision received November $12,1973$. ) 